\title{
Lessons From Spider and Silkworm Silk Guts
}

\author{
José Pérez-Rigueiro 1,2,3*, Víctor Ruiz"1,2, José Luis Cenis ${ }^{4}$, Manuel Elices ${ }^{2}$ and \\ Gustavo Víctor Guinea ${ }^{1,2,3}$ \\ ${ }^{1}$ Centro de Tecnología Biomédica, Universidad Politécnica de Madrid, Madrid, Spain, ${ }^{2}$ Departamento de Ciencia \\ de Materiales, ETSI Caminos, Canales y Puertos, Universidad Politécnica de Madrid, Madrid, Spain, ${ }^{3}$ Biomedical Research \\ Networking Center in Bioengineering, Biomaterials and Nanomedicine (CIBER-BBN), Madrid, Spain, ${ }^{4}$ Departamento \\ de Biotecnología, Instituto Murciano de Investigación y Desarrollo Agrario y Alimentario (IMIDA), Murcia, Spain
}

The results presented in previous works on the formation of fibers from silkworm and spider silk guts are reviewed and new information is included on the glands that allow the formation of these fibers. Silk gut fibers are obtained directly from the silk glands by immersion in a mild acid solution and subsequent stretching. The fibers so produced were characterized in terms of their microstructure and mechanical properties. The comparison of silk gut fibers with their natural counterparts allows gaining new insights in the processing, microstructure and properties of both silkworm and spider silks.

Keywords: biomimetics, mechanical characterization, silkworm silk, spider silk, spinning

OPEN ACCESS

Edited by:

Nicola Maria Pugno, University of Trento, Italy

Reviewed by:

Giuseppe Puglisi, Politecnico di Bari, Italy Domenico De Tommasi, Politecnico di Bari, Italy

*Correspondence: José Pérez-Rigueiro jose.perez@ctb.upm.es

Specialty section: This article was submitted to Mechanics of Materials, a section of the journal

Frontiers in Materials

Received: 09 September 2019 Accepted: 12 February 2020

Published: 28 February 2020

Citation:

Pérez-Rigueiro J, Ruiz V, Cenis JL, Elices M and Guinea GV (2020)

Lessons From Spider and Silkworm

Silk Guts. Front. Mater. 7:46.

doi: 10.3389/fmats.2020.00046

\section{INTRODUCTION}

Silkworm and spider silks represent a fascinating biological system at the frontier between Materials Science, Biology and Biotechnology (Heim et al., 2009). In spite of originating from two independent evolutionary events (Craig and Riekel, 2002), the concurrence of many of their defining features points to the robustness and universality of their design principles. In this regard, both systems are based on the conversion of a protein solution into high performance solid fibers under extremely mild processing conditions (Vollrath and Knight, 2001; Jin and Kaplan, 2003).

Consequently, the study of silks and the development of artificial processes and new materials inspired in these fibers has represented one of the major driving forces in the field of biomimetics (Schmitt, 1969). The implementation of the biomimetic approach, however, requires unveiling the basic principles of the natural system that, in the case of silks, is hampered by the subtle interplay of all the contributing elements to the spinning process. Thus, discriminating between the basic principles of the process and those accidental to couple the system with the living spinning organism, either worm or spider, proved a most difficult task. As a result of this difficulty and although significant advances were made on the understanding of the system (Iizuka, 1985; Askarieh et al., 2010; Hagn et al., 2010), the development of biomimetic spinning systems and fibers is still an intensive area of research (Madurga et al., 2017).

In this context, the possibility of producing fibers directly from the silk glands offers a clear opportunity to explore the elements of the process following a route that differs from the natural spinning system. The fibers produced from the silk glands, known as silk gut fibers or, briefly, silk guts ("hijuelas" in Spanish and "crins de Florence" in French) were a traditional craftsmanship product in southern Europe, especially in the Región de Murcia (Spain), during the XVIII and XIX centuries (Humphries, 1949; Marden, 1951; Martin, 1991). Silkworm silk gut fibers were produced by immersing the worms in a vinegar solution and by subsequently stretching the glands, leading 
to what were the best fibers of its time. The production of silk guts was discontinued in the 1950s due to the impossibility to compete in the market with the new polymeric fibers, such as nylon.

In spite of its long history, no detailed characterization of the microstructure and mechanical behavior of silkworm silk gut was available until 2015 (Cenis et al., 2015), when it was found that the comparison of the silk guts with the natural materials allowed a deep insight on the spinning process and on the microstructure and mechanical properties of both materials. In addition, it was also found that a similar process leads to the formation of spider silk gut fibers from spider silk glands (Jiang et al., 2014; Ruiz et al., 2019). This work reviews the main findings obtained from the comparison of silkworm and spider silk gut fibers with their natural counterparts, and highlights those aspects deemed more relevant for the study of these material from a biomimetic perspective.

\section{EXPERIMENTAL WORK}

The details on the experimental work on the formation of silkworm and spider silk guts and their subsequent microstructural and mechanical characterization are provided elsewhere (Jiang et al., 2014; Cenis et al., 2015; Ruiz et al., 2019). Briefly, glands were retrieved from the spinning organisms after being anesthetized. Silk gland extraction is straightforward with worms, but requires the careful assessment of the gland to be used when working with spiders. This assessment was performed through mass spectroscopy by identifying peptides characteristics of each spidroin as found from Genetic analysis (Babb et al., 2017). After being retrieved, the glands were immersed in Ringer's solution for a period no longer than $30 \mathrm{~min}$. Glands were then submerged into a mild acetic acid solution, typically with a concentration in the range $1-5 \%$, and removed after a period of time between 1 and $6 \mathrm{~min}$. The excess of water was gently removed and the glands were stretched either with a tensile testing machine or by hand.

Stretching the gland in a tensile testing machine allows measuring the forces that appear during the formation of the silk gut. These forces were converted to engineering stress vs. engineering strain curves. The engineering strain sustained by the gland during the formation process was defined as the increment in length of the gland divided by the initial length of the gland (typically $60 \mathrm{~mm}$ for silkworm silk and $5 \mathrm{~mm}$ for major ampullate gland silk). The engineering stress to which the gland is subjected during the formation process of the silk gut fiber were calculated as the ratio between the measured force and the maximum cross sectional area of the silk gut as measured in an optical or scanning electron microscope. Usage of the maximum cross sectional area in this case corresponds to calculating the minimum stress required for completing the transition of protein solution into solid fiber.

Silk guts were mounted on aluminum foil frames with gauge lengths between 10 and $60 \mathrm{~mm}$, and micrographs were obtained either in an optical microscope or in a scanning electron microscope in order to determine the apparent diameter of the fibers. The cross sectional area was calculated from the apparent diameter of the silk gut by assuming a circular cross section. Engineering stresses of the silk gut fibers were calculated by taking the minimum diameter of the silk gut, and true stresses were calculated from engineering stresses under the assumption of volume constancy (Guinea et al., 2006). In this case, the usage of the minimum cross sectional area of the silk gut fiber is intended to provide the value for the maximum stress that the fiber may sustain. The microstructural characterization of the silk guts was performed through Fourier transform infrared spectroscopy (FTIR) and X-ray diffraction (XRD).

\section{RESULTS AND DISCUSSION}

\section{Silk Guts From Silkworm and Spider Silk Glands}

The historical process for making fibers from silk guts was developed with silkworms and thrust by the practical applications of the material. Silkworm silk guts were formed traditionally in a two-step process that required immersion in a vinegar solution and subsequent manual stretching (Humphries, 1949; Marden, 1951). An example of a fiber obtained following this process, although the vinegar solution is substituted by an acetic acid solution, is shown in Figure 1A. Extending this process to obtain fibers from silk guts from spider silk glands had to face with two main difficulties. Firstly, orb-web spiders present up to seven different silk glands, from which up to five different types of fibers are spun (Heim et al., 2009). Since each fiber is endowed with a characteristic set of tensile properties (Blackledge and Hayashi, 2006), it was likely that spider silk guts produced from different glands might exhibit different properties. Consequently, it was necessary to get a proper identification of the glands being employed. Secondly, the size of spider silk glands is much smaller than that of silkworms, even if working with specimens from the Nephila genus, that includes some of the largest orb-web spiders. This smaller size renders the manipulation of the gland and, especially, the stretching step much more difficult than for silkworm silk gut. Fortunately, these drawbacks were overcome

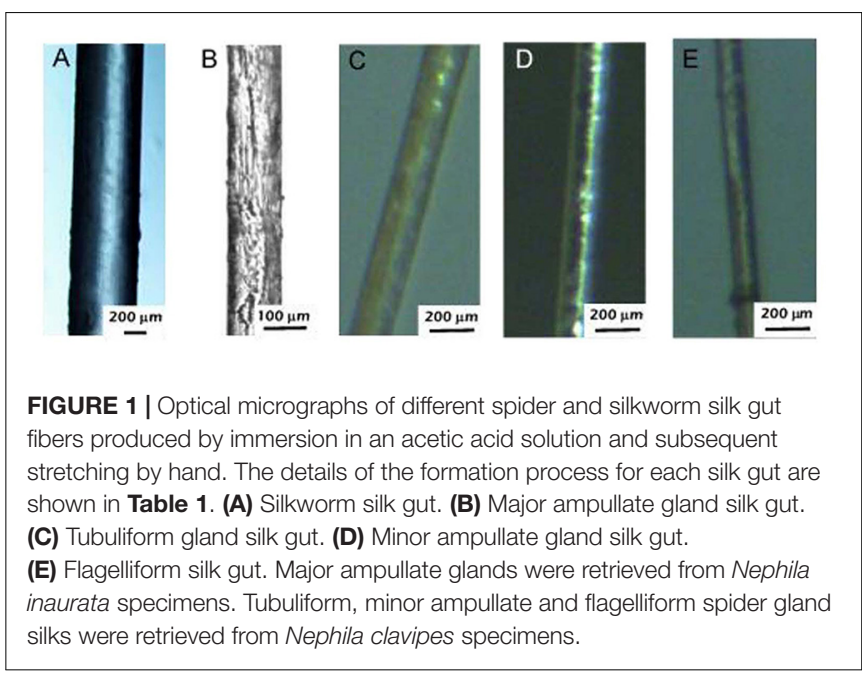


in most cases and Figures 1B-E show optical micrographs of spider silk guts obtained from the major ampullate gland, the tubuliform gland, the minor ampullate gland and the flagelliform gland, respectively. All glands were retrieved from Nephila clavipes specimens, except for the major ampullate gland that was retrieved from a Nephila inaurata spider. Attempts to produce silk gut from the acciniform gland failed due its small size and the impossibility to stretch the gland after being immersed in the acetic acid solution. The detailed conditions for the formation of each silk gut shown in Figure $\mathbf{1}$ are indicated in Table $\mathbf{1}$.

The possibility of producing fibers from silk guts of all the spider silk glands amenable to being manipulated suggest the existence of a common spinning process that is likely to have appeared in the ancestral silk gland (Blackledge et al., 2009). In this regard, it is more surprising that a similar process leads to the formation of silkworm silk glands. Silkworm and spider silks are thought to be the result of two independent evolutionary events. Consequently, the parallelism in the processing of both materials may be understood as an indication of the robustness of a process that allows the conversion of a protein solution into high performance solid fibers. As indicated above, the difficulty in obtaining silk guts increases when the size of the gland decreases. Consequently, the most complete microstructural and mechanical characterization of silk guts is limited up to date to the larger glands: silkworm silk, and spider major ampullate and tubuliform glands. For this reason, these are the materials on which the discussion below will be based.

\section{Silkworm Silk and Silkworm Silk Gut Fibers}

The tensile properties of natural silkworm and silk gut fibers are compared in Figure 2. Since the properties of natural silkworm silk depend on the treatment that the material undergoes before being tested, representative true stress-true strain curves of fibers either obtained by forced silking or subjected to a subsequent degumming treatment are presented. Both conditions were shown to summarize the large variability in the tensile properties that is characteristic of the natural material (Belen Perea et al., 2016). Although the qualitative mechanical behavior of all fibers is comparable, there are significant quantitative differences between silk gut fibers and natural fibers that preclude the identification of the former with their natural counterparts. In other words, the silk gut formation process applied to silkworm

TABLE 1 | Processing conditions of the silk guts shown in Figure 1.

\begin{tabular}{lcc}
\hline Silk gland & $\begin{array}{c}\text { Acetic acid } \\
\text { concentration (\%) }\end{array}$ & $\begin{array}{c}\text { Immersion } \\
\text { time (min) }\end{array}$ \\
\hline Silkworm (Bombyx mori) & 2 & 2 \\
Major ampullate gland (Nephila inaurata) & 1 & 3 \\
Tubuliform gland (Nephila clavipes) & 1 & 1 \\
Minor ampullate gland (Nephila clavipes) & 1 & 1 \\
Flagelliform gland (Nephila clavipes) & 5 & $3(+2)$
\end{tabular}

Flagelliform silk gut was formed after immersion in the acetic acid solution for 3 min, and stretched 2 min later after being removed from the acetic acid solution.

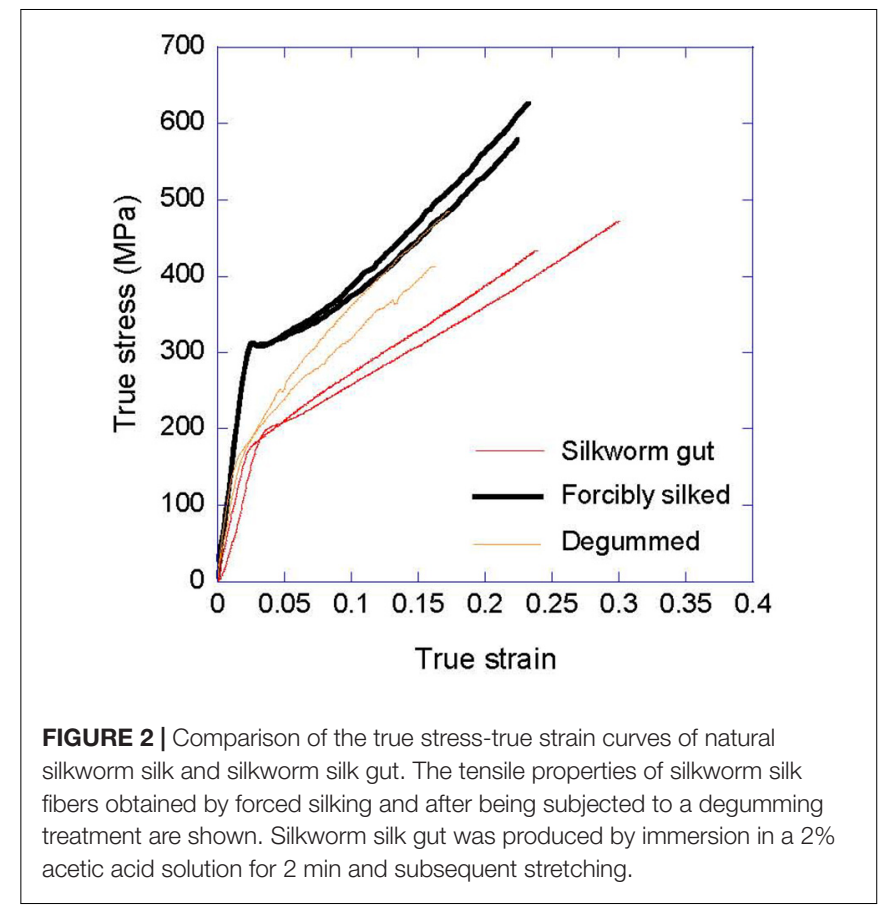

silk glands does not lead to the formation of proper silkworm silk, so that the differences between silkworm natural silk, and silk gut are deeper than the mere variation in the cross sectional area of the fibers obtained by both processing methods. Differences are observed very clearly when comparing the elastic modulus of the natural silk, that ranges between $E=14 \mathrm{GPa}$ (forcibly silked) and $E=12 \mathrm{GPa}$ (degummed) for the natural material, and the value of $E=7 \mathrm{GPa}$, measured for silkworm silk gut. Differences are also apparent when the regions at high strains beyond the yield point are compared. In this region the average slope of the silkworm silk gut stress-strain curves is significantly smaller than those of the natural silk fibers, either forcibly silked or after being subjected to degumming.

The XRD analysis of silkworm silk gut has revealed some microstructural features that justify the differences in the mechanical behavior observed between natural fibers and silk gut fibers. Representative XRD patterns of natural silkworm silk and silkworm silk gut fibers are presented in Figures 3A,B, respectively. It was found that both materials share a common unit cell, but significant variations are observed in terms of crystallinity and in the size of the $\beta$-nanocrystals. Thus, crystallinity in silkworm silk gut reaches a value of $\sim 30 \%$, that is half of the value found in silkworm silk. This reduction in crystallinity accounts for the reduction in the elastic modulus of silkworm silk gut fibers compared with the natural fibers. In addition, a two-state polymer model (Puglisi et al., 2017) may account for the reduction in the average slope of the silkworm silk gut stress-strain curve in the high strain region when compared with the natural material. The two-state polymer model assumes that the micromechanism responsible for the deformation of the polymeric fiber is the transition from rigid folded to entropic folded domains. The higher crystallinity of the natural silk 


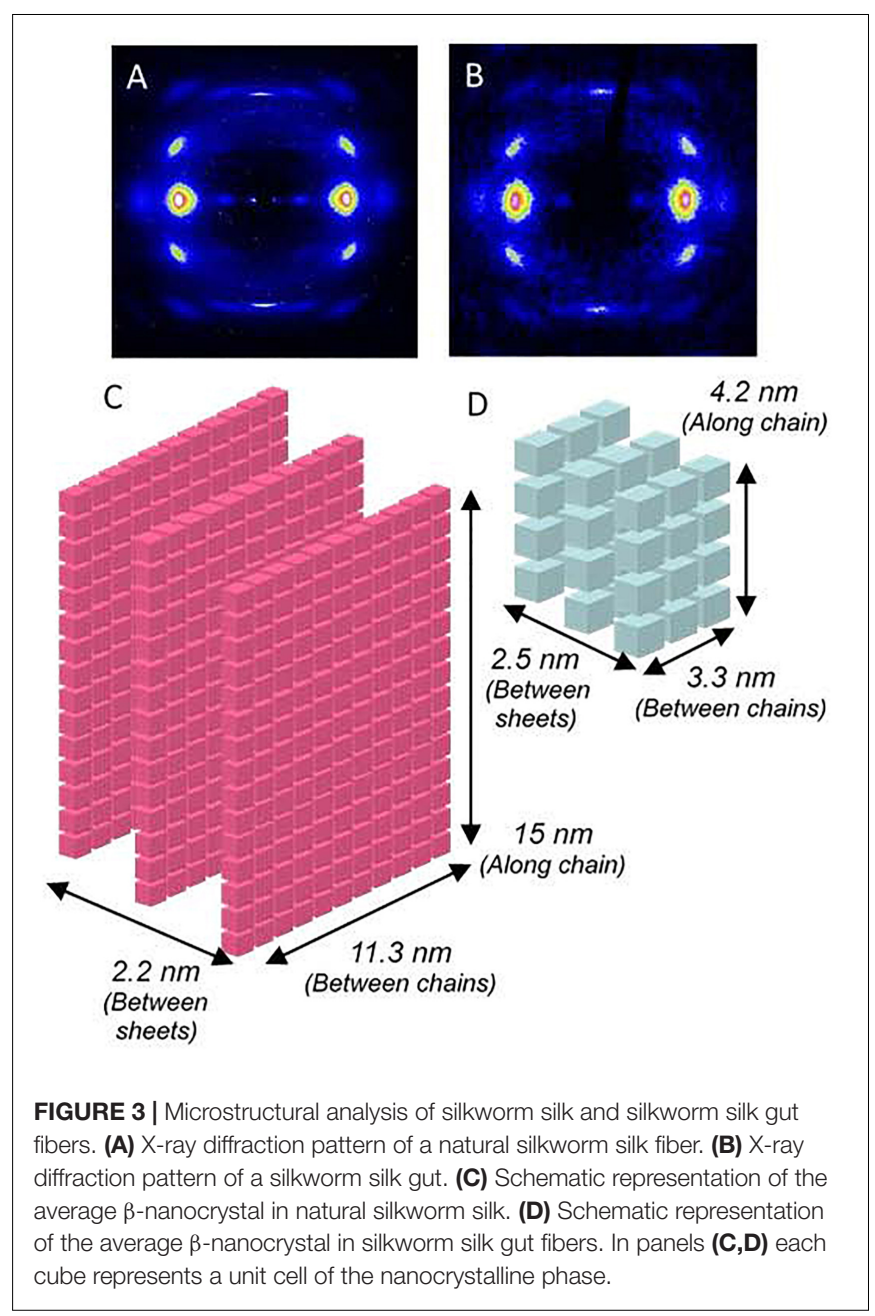

fibers implies the existence of a larger fraction of rigid folded domains, whose unfolding requires higher values of stress, as observed experimentally.

The differences between the natural material and silk guts are even more significant when the size of the nanocrystals is considered. A schematic representation of the $\beta$-nanocrystals as found in silkworm silk and silk gut is shown in Figures $3 C$,D, in which each cube represents a unit cell. From these schemes it is apparent that the volume of a $\beta$-nanocrystal in natural silkworm silk is much larger than that of a $\beta$-nanocrystal in silk gut. This result suggests that the appearance of the highly crystalline structure of silkworm silk is dependent on the details of the natural spinning process in the worm. Some of the processing conditions must not be reproduced in the process that leads to the formation of the silk gut fibers and, consequently, the microstructure of the latter does not reach such a high value for the crystalline fraction. The deviation of the natural silkworm silk fiber cross section from a circular shape might be a clue, since it indicates that the fiber must be subjected to significant shear stresses during its processing in the silk gland.

Another interesting aspect raised by the comparison of the $\beta$-nanocrystals in natural silk and silk guts is the formation of the nanocrystals from the piling up of exactly three $\beta$-pleated sheets in both cases. The persistence of this microstructural feature in contrast with the large overall differences between the nanocrystalline phases in both materials might reflect a deep design principle of silkworm silk. A similar piling up of exactly three $\beta$-pleated sheets that make up the nanocrystalline phase was also found in an extensive analysis of the microstructure of MA spider silk retrieved from different species (Madurga et al., 2015).

\section{Major Ampullate and Tubuliform Silks and Silk Guts}

In spite of the parallelisms in the formation process of silkworm and spider silk guts, the comparison of the latter with natural spider silk fibers points to the existence of profound divergences in some design principles between silk spinning in worms and spiders. As indicated above, although spider silk gut fibers were obtained from four different spider silk glands: major ampullate gland, tubuliform gland, minor ampullate gland, and flagelliform gland, only silk guts produced from the two largest glands (MA and Tub) were amenable for a detailed mechanical and microstructural characterization. The comparison of MA and Tub silk guts with their natural counterparts is presented in Figures 4A,B.

Figure 4A compares the tensile properties of natural MA silk and MA silk gut fibers obtained from $N$. inaurata spiders. The large variability shown by MA silk, even when a single spider species is considered has represented a major challenge for the characterization of the material (Madsen et al., 1999; PerezRiguero et al., 2001). In this regard, the application of techniques based on the supercontraction of MA silk (Work, 1977; Perez-Rigueiro et al., 2003) allows a relatively straightforward procedure for the comparison of MA fibers spun under different experimental conditions or spun by different species (Madurga et al., 2016). Consequently, the possible supercontraction ability of MA silk gut fibers was assessed and it was found that, as with the natural material, MA silk gut fibers exhibit a ground state to which the fiber can revert by immersion in water. The existence of this ground state is considered as the defining property of a supercontracting fiber (Perez-Rigueiro et al., 2019).

From Figure 4A it is apparent that MA silk gut fibers subjected to maximum supercontraction concur with the natural MA fibers, also subjected to maximum supercontraction, up to the breaking point of the former. The similarity between both fibers was also supported by the concurrence of their FTIR spectra and XRD patterns (Jiang et al., 2014). Consequently, MA silk gut fibers can be identified with natural MA spider silk, so that the only essential difference between both fibers is the large divergence between their cross sectional areas. This difference in the cross sectional area might also account for the reduced tensile strength of MA silk gut fibers, since it is a well-established principle of Materials Science that tensile strength is inversely proportional to the cross sectional area of a fiber. Although this principle is mainly supported by data obtained from artificial materials, it is reasonable to assume that a larger area also allows the existence of larger defects in natural materials, leading to a reduced strength. In contrast with the extreme variability 

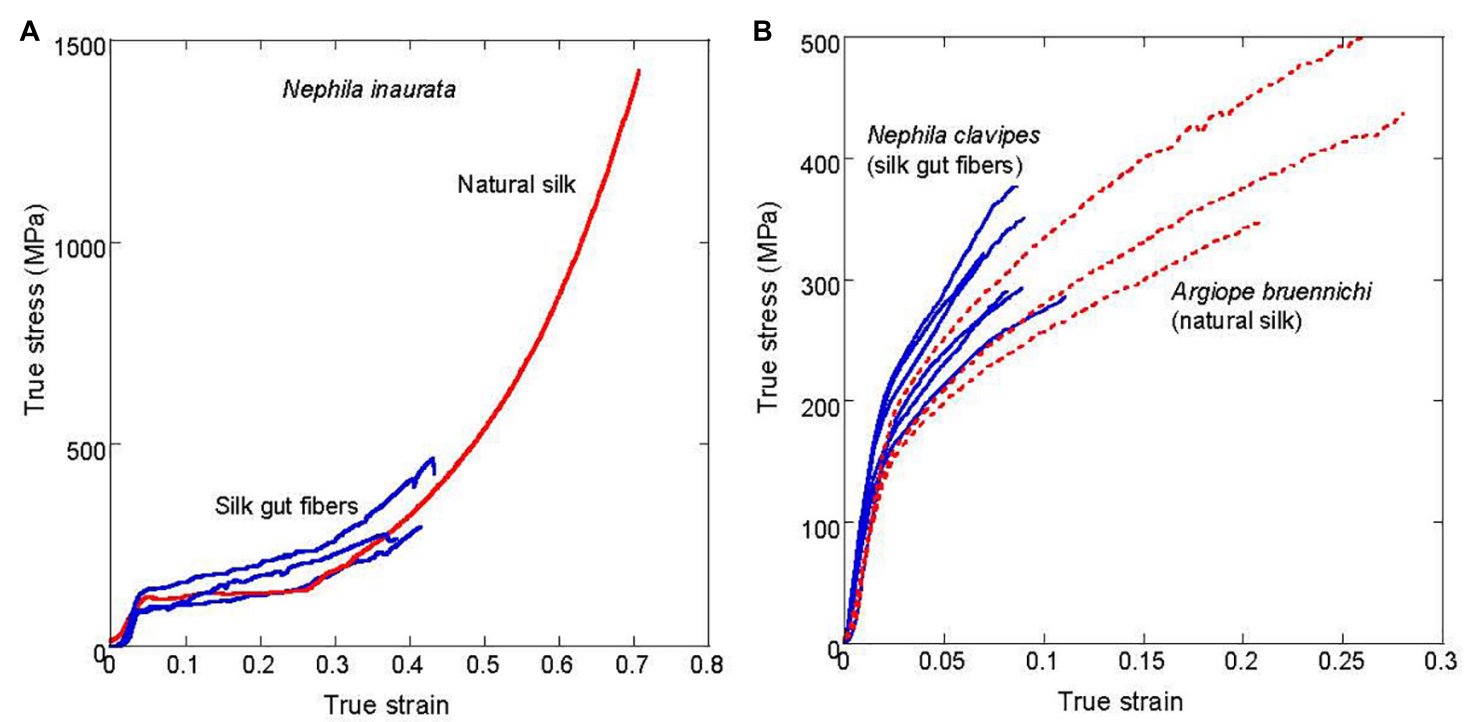

FIGURE 4 | Comparison of the true stress-true strain curves of natural spider silk and spider silk gut fibers. (A) Comparison of the major ampullate gland silk natural fibers and major ampullate silk gut fibers of Nephila inaurata. (B) Comparison of the tubuliform natural fibers spun by Argiope bruennichi spiders and tubuliform silk gut fibers produced from Nephila clavipes spiders.

characteristic of MA fibers, Figure 4B illustrates the extreme conservation of tubuliform silk. In this case, the tensile behavior of natural Tub silk and Tub silk gut fibers is shown to concur even when fibers from two species that diverged over 120 Mya (Elices et al., 2009) (Argiope bruennichi and N. clavipes) are compared.

The previous results highlight two profound principles in the design of the spider silk spinning system. First of all, it is apparent that the instructions required for forming the solid fiber from a protein solution are contained in the spidroin solution in the gland and are independent of the detailed spinning mechanism, in particular from the spider gland silk. It is found that the formation of spider silk gut fibers, either MA or Tub, only requires a change in the $\mathrm{pH}$ of the solution and subsequent stretching, and a material with the same microstructure and mechanical properties of the natural spun fibers is obtained. In addition, it also shows the intriguing contrast between the extreme variability observed in MA silk and the extreme conservation of Tub silk. In spite of sharing a common organization based on large proteins constituted by a small number of repetitive amino acid motifs, the large differences observed between both silk fibers opens the question of which evolutionary pressures may have led to these two divergent behaviors.

\section{Process of Silk Fibers Formation From a Protein Solution}

The capacity to produce silk gut fibers with properties comparable to their natural counterparts also allows access to some features of the transition between protein solution and solid fiber that are usually hidden in the silk glands of the spinning organisms. Thus, it is feasible to measure the forces (and stresses) involved in the formation of the silk gut fibers and, in addition, it is also possible to follow the early microstructural changes associated with the formation of the solid fiber.

Figure 5A illustrates the forces involved in the formation process of silkworm and MA silk gut fibers expressed as engineering stress-engineering strain curves. It is again striking the concurrence between both curves in spite of the differences in evolutionary origin between the spinning organisms. There are, however, some remarkable differences in the details of both curves, that point to differences in some of the deformation mechanisms of both silk guts. Thus, the recorded mechanical properties obtained during the formation MA silk do correspond to an elastomer (Gosline et al., 1984), and is consistent with the importance of the elastomeric behavior of the protein chains in the mechanical properties of MA silk (Termonia, 1994, 2000). In contrast, silkworm silk gut exhibits an initial stiffer region followed by a more compliant behavior at larger values of strain. A comparable tensile behavior was observed in silkworm (Bombyx mori) silk fibers tested in water (PerezRigueiro et al., 2000) and is not compatible with an elastomeric behavior of the protein chains in the initial steps of silkworm silk gut formation.

It was found that both glands were solidified at an engineering strain of $\sim 100 \%$ (i.e., the gland is stretched up to a length that doubles its initial length) and this value is shown on each curve by a solid circle. The combination of the stress-strain curve during the silk gut formation process and the observation that the fiber is formed at this value of strain allows determining the minimum force, or equivalently, the minimum stress required to complete the transition between protein solution and solid fiber. From Figure 5A a value of $\sim 1 \mathrm{MPa}$ is obtained, which is significantly lower than previous estimations on the stresses involved in the spinning of natural silks obtained from the rheological study of the protein solution (Holland et al., 2006; Kojic et al., 2006) or 

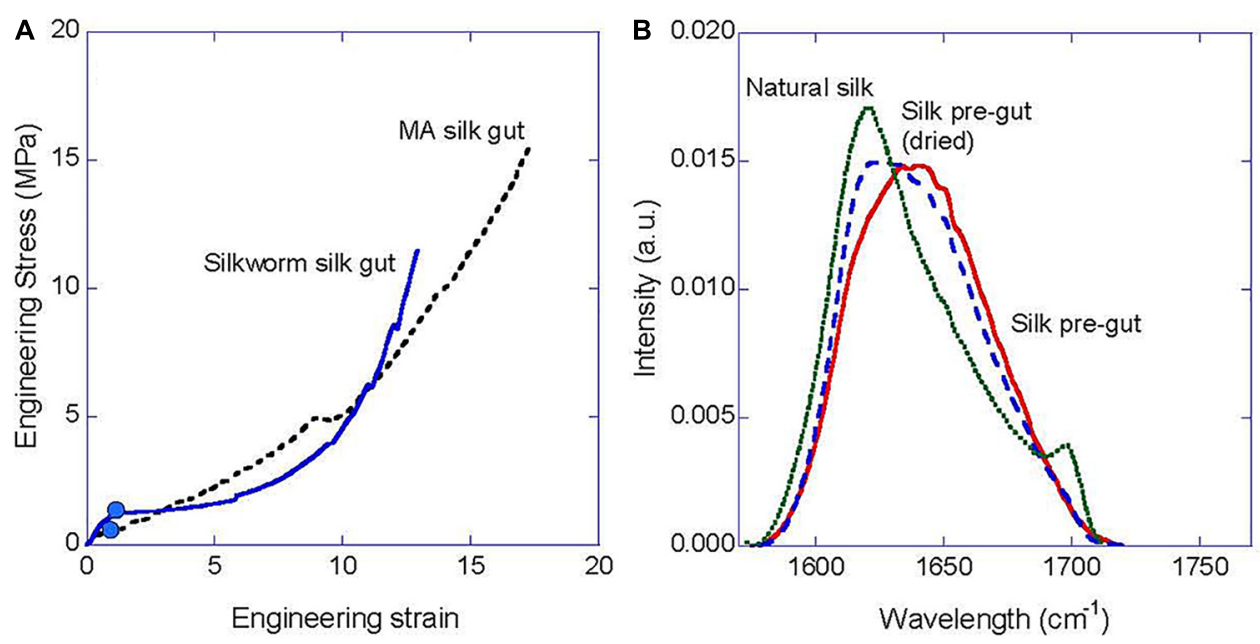

FIGURE 5 | Characterization of the silk gut formation process. (A) Illustrative engineering stress-engineering strain curve measured during the silk gut formation process from the gland of a silkworm (B. mori) and of a MA gland (N. inaurata). (B) Comparison of the FTIR spectra of natural B. mori silk fiber, a pre-gut fiber formed by stretching the gland up to a strain of $100 \%$, and a pre-gut fiber formed by stretching the gland up to a strain of $100 \%$ and being allowed to dry in air for $1 \mathrm{~h}$.

by measuring the forces exerted on the fiber during forced silking (Elices et al., 2006). Both experimental procedures had estimated a value of the spinning stresses between 20 and $40 \mathrm{MPa}$. The results obtained from the analysis of the forces involved in the formation of silk gut suggest that these larger values might be related with other processes in the spinning of the natural fiber [i.e., friction of the fiber with the valve in the spinning of MA silk (Ortlepp and Gosline, 2004)] different from the transition between protein solution to solid material.

Figure 5B shows the amide I peak of silkworm (B. mori) silk obtained by FTIR and compares it with the amide I peaks of a silkworm silk pre-gut (see below for the definition of pre-gut) and a silkworm silk pre-gut subjected to drying for $1 \mathrm{~h}$. Silkworm silk pre-gut was formed by stretching the gland up to an engineering strain of $e=100 \%$ (initial length of the gland: $60 \mathrm{~mm}$, final length: $120 \mathrm{~mm}$ ), stopping the process at that value of strain and recording the FTIR spectrum immediately. Alternatively, other pre-guts were produced under the same conditions and allowed to dry for $1 \mathrm{~h}$ before being characterized by FTIR. A clear shift of the maximum of the peak from $\sim 1640 \mathrm{~cm}^{-1}$ in the silk pregut, to $1620 \mathrm{~cm}^{-1}$ in the dried silk pre-gut and in the natural silkworm is observed. This shift is the result of the increasing contribution of $\beta$-pleated sheets to the secondary structure of the proteins (Pellerin et al., 2005). It is remarkable, however, that a significant fraction of $\beta$-pleated sheet is observed in the silk pre-gut fiber, which supports the hypothesis that the appearance of this secondary structure and, possibly, the piling up of the $\beta$-sheets into $\beta$-nanocrystals are very early events in the formation of the solid fiber. Drying of the pre-gut leads to an increase in the content of the $\beta$-pleated and to the formation of $3_{1}$ helices (Chen et al., 2001; Teramoto and Miyazawa, 2005), and illustrates the order in which the microstructure of silkworm silk is formed during the formation process of the fiber. A similar study was not possible on MA silk guts due to experimental difficulties related with the much smaller size of the MA gland $(\sim 5 \mathrm{~mm})$.

\section{CONCLUSION}

Silk gut fibers can be produced from the silk glands of silkworm (B. mori) and from the major ampullate gland silk, tubuliform gland silk, minor ampullate gland silk, and flagelliform gland silk of Nephila spiders by immersion of the glands in a mild acidified solution and subsequent stretching.

The stress-strain curves of silkworm silk gut fibers are qualitatively similar to those of the natural silkworm silk fibers, but differ in the value of the mechanical parameters, such as the elastic modulus. These differences can be traced back to variations in the crystalline phase between both materials. Crystallinity of silkworm silk gut fibers is half of that measured from the natural fibers. Besides, the size of the $\beta$-nanocrystals in silkworm silk gut fibers is much smaller than that of native silk. However, in spite of the differences between the crystalline phases of both materials, there is a persistence in the number of $\beta$-pleated sheets that form the nanocrystals, suggesting that this might be a design principle in the formation of the fiber.

In contrast to silkworm silk, MA and Tub spider silk gut fibers are shown to be the same material as their natural counterparts. MA spider silk gut fibers are endowed with the ability to supercontract and their properties concur with those of the natural material if both, silk gut fibers and native silk, are retrieved from the same species. The concurrence of the tensile behavior of Tub silk gut fibers and Tub native fibers of different species points to the persistence of these silk fibers throughout evolution. The identification of spider silk gut fibers and native fibers in this case indicates that the processing instructions for forming the fibers from the protein solution are contained in the spidroins and are independent of the natural gland system.

The possibility of producing silk gut fibers also offers a unique opportunity to monitor the early events leading to the formation of the fiber. In this regard, it is found that the major microstructural change leading to the formation of the solid 
material is the appearance of $\beta$-pleated secondary structure at a strain of $\sim 100 \%$. In addition, it is also found that the mechanical stresses required to complete the transition from the protein solution to the fibers are in the range of $\sim 1 \mathrm{MPa}$, which is a much smaller value than previous estimations suggested. Differences between these previous values and the one found from the analysis of the silk might arise from other forces that appear in the natural spinning process, but which are not directly involved in the liquid-solid transition. All these findings highlight the insights that can be gained from the comparison of silk guts and natural silk fibers and should encourage the making of new studies that might follow this rationale in the future.

\section{DATA AVAILABILITY STATEMENT}

The datasets generated for this study are available on request to the corresponding author.

\section{REFERENCES}

Askarieh, G., Hedhammar, M., Nordling, K., Saenz, A., Casals, C., Rising, A., et al. (2010). Self-assembly of spider silk proteins is controlled by a $\mathrm{pH}$-sensitive relay. Nature 465, 236-239. doi: 10.1038/nature08962

Babb, P. L., Lahens, N. F., Correa-Garhwal, S. M., Nicholson, D. N., Kim, E. J., Hogenesch, J. B., et al. (2017). The Nephila clavipes genome highlights the diversity of spider silk genes and their complex expression. Nat. Genet. 49, 895-903. doi: 10.1038/ng.3852

Blackledge, T. A., and Hayashi, C. Y. (2006). Silken toolkits: biomechanics of silk fibers spun by the orb web spider Argiope argentata (Fabricius 1775). J. Exp. Biol. 209, 2452-2461. doi: 10.1242/jeb.02275

Blackledge, T. A., Scharff, N., Coddington, J. A., Szuts, T., Wenzel, J. W., Hayashi, C. Y., et al. (2009). Reconstructing web evolution and spider diversification in the molecular era. Proc. Natl. Acad. Sci. U.S.A. 106, 5229-5234. doi: 10.1073/ pnas.0901377106

Cenis, J. L., Madurga, R., Aznar-Cervantes, S. D., Abel Lozano-Perez, A., Mari-Buye, N., Meseguer-Olmo, L., et al. (2015). Mechanical behaviour and formation process of silkworm silk gut. Soft Matter 11, 8981-8991. doi: 10.1039/ c5 $5 \mathrm{sm} 01877 \mathrm{c}$

Chen, X., Knight, D. P., Shao, Z. Z., and Vollrath, F. (2001). Regenerated Bombyx silk solutions studied with rheometry and FTIR. Polymer 42, 99699974.

Craig, C. L., and Riekel, C. (2002). Comparative architecture of silks, fibrous proteins and their encoding genes in insects and spiders. Comp. Biochem. Physiol. B Biochem. Mol. Biol. 133, 493-507. doi: 10.1016/s1096-4959(02) 00095-7

Elices, M., Guinea, G. V., Plaza, G. R., Real, J. I., and Perez-Rigueiro, J. (2006). Example of microprocessing in a natural polymeric fiber: role of reeling stress in spider silk. J. Mater. Res. 21, 1931-1938. doi: 10.1557/jmr.2006. 0240

Elices, M., Plaza, G. R., Arnedo, M. A., Pérez-Rigueiro, J., Torres, F. G., and Guinea, G. V. (2009). Mechanical Behaviour of Silk during the evolution of Orbweb spinning spiders. Biomacromolecules 10, 1904-1910. doi: 10.1021/bm900 $312 \mathrm{c}$

Gosline, J. M., Denny, M. W., and Demont, M. E. (1984). Spider silk as rubber. Nature 309, 551-552. doi: 10.1038/309551a0

Guinea, G. V., Perez-Rigueiro, J., Plaza, G. R., and Elices, M. (2006). Volume constancy during stretching of spider silk. Biomacromolecules 7, 2173-2177. doi: $10.1021 / \mathrm{bm} 060138 \mathrm{v}$

Hagn, F., Eisoldt, L., Hardy, J. G., Vendrely, C., Coles, M., Scheibel, T., et al. (2010). A conserved spider silk domain acts as a molecular switch that controls fibre assembly. Nature 465, 239-242. doi: 10.1038/nature08936

\section{AUTHOR CONTRIBUTIONS}

VR prepared the spider silk guts. JP-R and JC prepared the silkworm silk guts and coordinated the research. All authors have discussed the results and revised the manuscript.

\section{FUNDING}

This work was funded by the Ministry of Economy and Competitiveness in Spain through projects MAT2016-75544C2 and MAT2016-79832-R, and Comunidad de Madrid (Spain) through grants NEUROCENTRO-B2017/BMD-3760 and IND2018/BMD-9804.

\section{ACKNOWLEDGMENTS}

Spiders were reared in Reptil Madrid S.L. by Oscar Campos.

Heim, M., Keerl, D., and Scheibel, T. (2009). Spider silk: from soluble protein to extraordinary fiber. Angew. Chem. Int. Ed Engl. 48, 3584-3596. doi: 10.1002/ anie. 200803341

Holland, C., Terry, A. E., Porter, D., and Vollrath, F. (2006). Comparing the rheology of native spider and silkworm spinning dope. Nat. Mater. 5, 870-874. doi: $10.1038 /$ nmat 1762

Humphries, A. M. C. (1949). The story of silk and silkworm gut. Post Grad. Med. J 25, 483-488. doi: 10.1136/pgmj.25.288.483

Iizuka, E. (1985). Silk thread - mechanism of spinning and its mechanicalproperties. Appl. Polym. Symp. 41, 173-185.

Jiang, P., Mari-Buye, N., Madurga, R., Arroyo-Hernandez, M., Solanas, C., GananCalvo, A., et al. (2014). Spider silk gut: development and characterization of a novel strong spider silk fiber. Sci. Rep. 4, 7326. doi: 10.1038/srep07326

Jin, H. J., and Kaplan, D. L. (2003). Mechanism of silk processing in insects and spiders. Nature 424, 1057-1061. doi: 10.1038/nature01809

Kojic, N., Bico, J., Clasen, C., and McKinley, G. H. (2006). Ex vivo rheology of spider silk. J. Exp. Biol. 209, 4355-4362. doi: 10.1242/jeb.02516

Madsen, B., Shao, Z. Z., and Vollrath, F. (1999). Variability in the mechanical properties of spider silks on three levels: interspecific, intraspecific and intraindividual. Int. J. Biol. Macromol. 24, 301-306. doi: 10.1016/s01418130(98)00094-4

Madurga, R., Blackledge, T. A., Perea, G. B., Plaza, G. R., Riekel, C., Burghammer, M., et al. (2015). Persistence and variation in microstructural design during the evolution of spider silk. Sci. Rep. 5:14820. doi: 10.1038/srep14820

Madurga, R., Ganan-Calvo, A. M., Plaza, G. R., Guinea, G. V., Elices, M., and PerezRigueiro, J. (2017). Production of high performance bioinspired silk fibers by straining flow spinning. Biomacromolecules 18, 1127-1133. doi: 10.1021/acs. biomac.6b01757

Madurga, R., Plaza, G. R., Blackledge, T. A., Guinea, G. V., Elices, M., and PerezRigueiro, J. (2016). Material properties of evolutionary diverse spider silks described by variation in a single structural parameter. Sci. Rep. 6:18991. doi: 10.1038/srep18991

Marden, L. (1951). Spain's silkworm gut. Natl. Geogr. Mag. 100, 100-108.

Martin, L. H. H. (1991). The history of silkworm gut. Am. Fly Fish. 17, 3-7.

Ortlepp, C., and Gosline, J. (2004). Consequences of forced silking. Biomacromolecules 5, 727-731. doi: 10.1021/bm034269x

Pellerin, C., Rousseau, M. E., Cote, M., and Pezolet, M. (2005). Study of molecular orientation by vibrational spectroscopy: from polymers to silk. Macromol. Symp. 220, 85-98. doi: 10.1002/masy.200550207

Perea, G. B., Solanas, C., Mari-Buye, N., Madurga, R., Agullo-Rueda, F., Muinelo, A., et al. (2016). The apparent variability of silkworm (Bombyx mori) silk and its relationship with degumming. Eur. Polym J. 78, 129-140. doi: 10.1016/j. eurpolymj.2016.03.012 
Perez-Rigueiro, J., Elices, M., and Guinea, G. V. (2003). Controlled supercontraction tailors the tensile behaviour of spider silk. Polymer 44, 3733-3736. doi: 10.1016/s0032-3861(03)00245-3

Perez-Rigueiro, J., Madurga, R., Ganan-Calvo, A. M., Elices, M., Guinea, G., Tasei, Y., et al. (2019). Emergence of supercontraction in regenerated silkworm (Bombyx mori) silk fibers. Sci. Rep. 9:2398. doi: 10.1038/s41598-019-38712-6

Perez-Rigueiro, J., Viney, C., Llorca, J., and Elices, M. (2000). Mechanical properties of silkworm silk in liquid media. Polymer 41, 8433-8439. doi: 10.1016/s00323861(00)00179-8

Perez-Riguero, J., Elices, M., Llorca, J., and Viney, C. (2001). Tensile properties of Argiope trifasciata drag line silk obtained from the spider's web. J. Appl. Polym. Sci. 82, 2245-2251. doi: 10.1002/app.2072

Puglisi, G., De Tommasi, D., Pantano, M. F., Pugno, N. M., and Saccomandi, G. (2017). Micromechanical model for protein materials: from macromolecules to macroscopic fibers. Phys. Rev. E 96:042407. doi: 10.1103/PhysRevE.96.042407

Ruiz, V., Jiang, P., Mueller, C., Jorge, I., Vazquez, J., Ridruejo, A., et al. (2019). Preparation and characterization of Nephila clavipes tubuliform silk gut. Soft Matter 15, 2960-2970. doi: 10.1039/c9sm00212j

Schmitt, O. H. (1969). "Some interesting and useful biomimetic transforms," in Proceedings of the Third International Biophysics Congress, Boston, MA, 297.

Teramoto, H., and Miyazawa, M. (2005). Molecular orientation behavior of silk sericin film as revealed by ATR infrared spectroscopy. Biomacromolecules 6, 2049-2057. doi: 10.1021/bm0500547
Termonia, Y. (1994). Molecular modeling of spider silk elasticity. Macromolecules 27, 7378-7381. doi: 10.1021/ma00103a018

Termonia, Y. (2000). "Molecular modelling of the stress/strainbehaviour of spider dragline," in Structural Biological Materials, ed. M. Elices (Amsterdam: Pergamon Press), 335-349.

Vollrath, F., and Knight, D. P. (2001). Liquid crystalline spinning of spider silk. Nature 410, 541-548. doi: 10.1038/35069000

Work, R. W. (1977). Dimensions, birefringences, and force-elongation behavior of major and minor ampullate silk fibers from orb-web-spinning spiders effects of wetting on these Properties. . Text. Res. J. 47, 650-662. doi: 10.1177/ 004051757704701003

Conflict of Interest: The authors declare that the research was conducted in the absence of any commercial or financial relationships that could be construed as a potential conflict of interest.

Copyright (c) 2020 Pérez-Rigueiro, Ruiz, Cenis, Elices and Guinea. This is an openaccess article distributed under the terms of the Creative Commons Attribution License (CC BY). The use, distribution or reproduction in other forums is permitted, provided the original author(s) and the copyright owner(s) are credited and that the original publication in this journal is cited, in accordance with accepted academic practice. No use, distribution or reproduction is permitted which does not comply with these terms. 